\title{
Investigation of Ar Ion-Milling Rates for Transmission Electron Microscopy Specimens
}

\author{
Min-Hee Lee, Kyou-Hyun Kim*
}

Advanced Process and Materials R\&BD Group, Incheon Regional Division, Korea Institute of Industrial Technology, Incheon 406-840, Republic of Korea

Recently, focused ion beam (FIB) is widely used to prepare a TEM specimen because the region of interest becomes narrow and specific. FIB system can precisely mill the specific area with a spatial accuracy of within few tens of nanometers. FIB, however, has problems such as Ga-induced damages near a sample surface which affect the investigation of original sample structure [1]. In addition, the thickness of TEM specimens prepared by FIB is commonly thicker than Ar-ion milled TEM specimens which makes difficult to obtain high quality of structural and chemical information from electron diffraction (ED) pattern, high resolution (HR) image, atomic resolution scanning-TEM (STEM) image, electron energy loss spectroscopy (EELS), etc. This then gives rise to the motivation that FIB-prepared specimens need to be further treated to reduce the artifacts induced by the high energy of Ga-ion sauce.

By contrast with FIB, the typical Ar-ion milling system uses much lower accelerating voltage and shows no implantation effect from Ar-ion. The recent Ar-ion milling systems provide rocking mode or selective sector speed thinning methods to mill TEM specimens uniformly down to few nanometers in thickness. Also, the damage from Ar-ion can be reduced by using the low accelerating voltage of $<1 \mathrm{kV}$. Based on the above, we applied the Ar-ion milling system to the FIB-prepared specimens in order to remove the damaged area induced by the Ga-ion sauce. Nevertheless, the FIB-prepared specimen is thin so that the Ar-ion milling rate needs to be monitored to prevent losing the specimen after the further milling using the Arion milling system. Motivated by the above considerations, we study here the Ar ion-milling rates for a couple of materials.

For this study, we selected the single crystals of $\mathrm{Si}$, GaAs, Ni and $\mathrm{Cu}$. The selected specimens are first pre-thinned up to $\sim 300 \mathrm{~nm}$ in thickness using FIB. In order to measure the Ar-ion milling rates, the FIB-prepared specimens were further Ar-ion milled at $1.5 / 3 \mathrm{kV}$ with a $6^{\circ}$ incident angle for 60 and 30 seconds, respectively. All samples were mounted on the center of Cu-grid as shown in Fig. 1. The Ar-ion beam directions are indicated by the arrows. The Arion milled specimens were then observed in TEM using convergent beam electron diffraction (CBED) to measure the thickness with help of Bloch simulation. For the Bloch simulation, we used the atomic scattering factors of Doyle and Turner [2], and the absorption parameters of Bird and King [3].

Figure 2(a) shows a medium magnification bright field (BF) image of Si single crystal. The BF image was recorded along the zone axis of [110]. The experimental CBED patterns for the Si single crystal were recorded from nine points as indicated in Fig. 2. Figures 2(b) and (c) respectively show the representative experimental CBED pattern and the corresponding simulated CBED pattern for the thickness of $279 \mathrm{~nm}$. The sample thickness can be then estimated as $279 \mathrm{~nm}$ from Figs. 2(b) and (c). This measurement is repeated to calculate the Ar-ion milling rates for Si, GaAs, Ni, and Cu. As shown in Figs. 2(b) and (c), the simulated CBED pattern well agrees with the experimental CBED pattern. We believe that this study provides a direct reference to estimate a milling time not only for the FIB-prepared specimens but also for the mechanically thinned specimens. 


\section{References}

[1] J. Mayer et al, MRS Bulletin 32 (2007), 400-407

[2] P. A. Doyle and P. S. Turner, Acta Crystallogr. Sect. A24 (1968), 390-397

[3] D. M. Bird and Q.A.King, ActaCrystallogr. Sect. A46 (1990), 202-208

[4] This work was supported by the research fund from Korea Institute of Industrial Technology (KITECH).

Figure 1. FIB-prepared TEM specimen mounted on a Cu-grid.
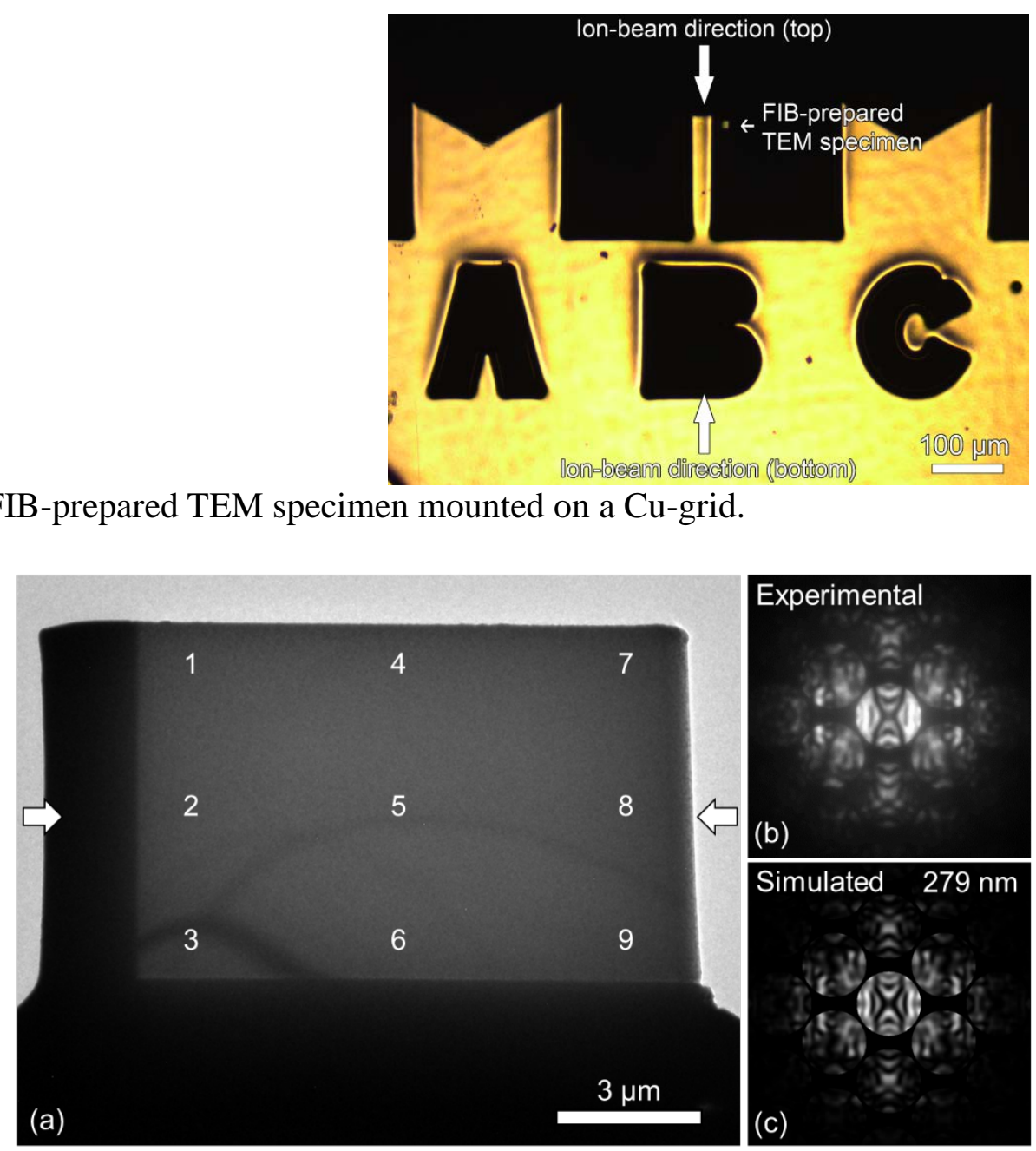

Figure 2. (a) A medium magnification image of Si single crystal prepared by FIB. (b) The experiment and (c) simulated CBED pattern to measure the thickñess of TEM specimen. 